\title{
The question of finding the shortest distance between two points of different diversity
}

\author{
Larisa Brylevskaya ${ }^{1 *}$, Larisa Gonchar $^{1}$, Elena Pastukhova $^{1}$, and Olga Skepko ${ }^{1}$ \\ ${ }^{1}$ Mining University of Saint Petersburg, 2, 21 st Line, St. Petersburg, 199106
}

\begin{abstract}
Digital education at the university implies application of information technologies, active learning methods and comprehensive algorithmizing of the process of solving various tasks. It is also supposed to create application software packages, which can find their application at the junction of various disciplines. (1) One area of digital education is to solve the problems of motion optimization and localization of various objects. In particular, the question of finding the shortest distance between points lying on two different varieties (curves, plane, etc.) is frequently solved (more than 10) Here are examples of the solving. The first example describes an algorithm for finding the shortest distance between two parabolas lying in the same plane; the second example suggests a solution course for an elliptical paraboloid and a point on the coordinate axis. Finally, the third example deals with the distance between a cube parabola and a hemisphere with the center at the beginning of the coordinates.
\end{abstract}

\section{Spacing of the two curves}

Suppose that on the plane in a rectangular Cartesian system of coordinate $O_{x y}$ are given two curves $l_{1}$ and $l_{2}$, which do not have common points and are described by the equations, respectively

$$
y_{1}=f\left(x_{1}\right), \quad y_{2}=g\left(x_{2}\right),
$$

where $\mathrm{f}(\mathrm{x})$ and $\mathrm{g}(\mathrm{x})$ functions are differentiated at some point $\mathrm{X}$. We will randomly choose 11 and $l_{2}$ curves a point $M_{1}\left(x_{1}, y_{1}\right)$ and $M_{2}\left(x_{2}, y_{2}\right)$ respectively. Then the length of the segment $M_{1} M_{2}$ can be calculated using the formula

$$
s\left(x_{1}, x_{2}\right)=\sqrt{\left(x_{2}-x_{1}\right)^{2}+\left(g\left(x_{2}\right)-f\left(x_{1}\right)\right)^{2}} .
$$

We will refer to the distance between $l_{1}$ and $l_{2}$ as the smallest value of function $s\left(x_{1}, x_{2}\right)$ assuming that $x_{1} \in X$ and $x_{2} \in X$

We will write out the necessary conditions of the extremum for the differentiated

\footnotetext{
*Corresponding author: brylev@mail.ru
} 
function of two variables $s\left(x_{1}, x_{2}\right)$

$$
\begin{aligned}
& \frac{\partial s}{\partial x_{1}}=\frac{x_{2}-x_{1}+\left(g\left(x_{2}\right)-f\left(x_{1}\right)\right) f^{\prime}\left(x_{1}\right)}{2 \sqrt{\left(x_{2}-x_{1}\right)^{2}+\left(g\left(x_{2}\right)-f\left(x_{1}\right)\right)^{2}}}=0, \\
& \frac{\partial s}{\partial x_{2}}=\frac{x_{2}-x_{1}+\left(g\left(x_{2}\right)-f\left(x_{1}\right)\right) g^{\prime}\left(x_{2}\right)}{2 \sqrt{\left(x_{2}-x_{1}\right)^{2}+\left(g\left(x_{2}\right)-f\left(x_{1}\right)\right)^{2}}}=0 .
\end{aligned}
$$

Assuming a pair of numbers $x_{1}^{k}, x_{2}^{k}$, where $k$ - is any natural number, indicates one of the solutions of the system of equations (3) and (4) corresponding to the minimum function $s\left(x_{1}^{k}, x_{2}^{k}\right)$. By choosing the lowest value from all minimums, we will obtain the required distance between curves $l_{l}$ and $l_{2}$, to which the values $x_{1}^{0}, x_{2}^{0}$, correspond if the interval $X$ is open. If the interval $X$ is closed, then we should calculate the values of the function $S\left(x_{1}, x_{2}\right)$ in the boundary points of the interval $X$ and discard the smallest of these numbers and allminimums.

From equations (3) and (4), it follows that

$$
f^{\prime}\left(x_{1}^{(0)}\right)=-\frac{1}{\frac{g\left(x_{2}^{(0)}\right)-f\left(x_{1}^{(0)}\right)}{x_{2}^{(0)}-x_{1}^{(0)}}}, \quad g^{\prime}\left(x_{2}^{(0)}\right)=-\frac{1}{\frac{g\left(x_{2}^{(0)}\right)-f\left(x_{1}^{(0)}\right)}{x_{2}^{(0)}-x_{1}^{(0)}}},
$$

firstly,

$$
f^{\prime}\left(x_{1}^{(0)}\right)=g^{\prime}\left(x_{2}^{(0)}\right)
$$

and, secondly, that the tangents to the $l_{1}$ and $l_{2}$ curve sat the points $M_{1}^{0}\left(x_{1}^{(0)}, f\left(x_{1}^{(0)}\right)\right)$ and $M_{2}^{0}\left(x_{2}^{(0)}, g\left(x_{2}^{(0)}\right)\right)$ respectively are orthogonal to the segment connecting these points, for the equation of a line passing through these points is as follows

$$
y-f\left(x_{1}^{(0)}\right)=\frac{g\left(x_{2}^{0}\right)-f\left(x_{1}^{0}\right)}{x_{2}^{(0)}-x_{1}^{(0)}}\left(x-x_{1}^{(0)}\right),
$$

Example. Consider curves $l_{1}$ and $l_{2}$ parabolas with the equations, respectively

$$
y_{1}=\frac{15}{572} x_{1}^{2}, \quad y_{2}=\frac{1}{4} x_{2}^{2}+\frac{1}{4} x_{2}+\frac{1573}{80} .
$$

According to equation (6) we could write

$$
x_{1}=\frac{143}{30}\left(2 x_{2}+1\right)
$$

Equation (4) in our case would look like the following 


$$
x_{2}-x_{1}+\left[\frac{1}{4} x_{2}^{2}+\frac{1}{4} x_{2}+\frac{1573}{80}-\frac{15}{572}\left(\frac{143}{30}\right)^{2}\left(2 x_{2}+1\right)^{2}\right]\left(\frac{1}{2} x_{2}+\frac{1}{4}\right)=0
$$

which, after identical transformations using equality (8), will be recorded as

$$
16 x_{2}^{3}+24 x_{2}^{2}-7 x_{2}=0
$$

Solving equation (9), we will get

$$
x_{2}^{(1)}=-\frac{7}{4}, \quad x_{2}^{(2)}=0, \quad x_{2}^{(3)}=\frac{1}{4} .
$$

Using the abscissa (10) points $M_{2}^{1}, M_{2}^{2}, M_{2}^{3}$ on the curve $l_{2}$ and the second equation in the pair (7), we can find the ordinates of these points and then we could write

$$
M_{2}^{1}=\left(-\frac{7}{4}, \frac{6397}{320}\right), \quad M_{2}^{2}=\left(0, \frac{1573}{80}\right), \quad M_{2}^{3}=\left(\frac{1}{4}, \frac{6317}{320}\right)
$$

Using value (10) and equality (8), we will find first an abscises of $M_{1}^{1}, M_{1}^{2}, M_{1}^{3}$ points on the curve $l_{l}$, and then with the first equality in the pair (7) their ordinates. As a result, we will get

$$
M_{1}^{1}=\left(-\frac{143}{12}, \frac{715}{192}\right), \quad M_{1}^{2}=\left(\frac{143}{30}, \frac{143}{240}\right), \quad M_{1}^{3}=\left(\frac{143}{20}, \frac{429}{320}\right)
$$

Using the formula (2) we will get that

$$
s\left(M_{1}^{1}, M_{2}^{1}\right)=19,208 ; \quad s\left(M_{1}^{2}, M_{2}^{2}\right)=19,583 ; \quad s\left(M_{1}^{3}, M_{2}^{3}\right)=19,669
$$

that means the distance between the parabolas $l_{1}$ and $l_{2}$ is 19,208.

\section{Distance determination from point to the plane}

We assume that in the rectangular Cartesian coordinate system $O x y z$ is given the point $A(\alpha, \beta, \gamma)$ and the ${ }^{\circ}$, defined by the equation $z=f(x, y)$, where the function $f(x, y)$ is differentiated in some area $D$ on the plane $O_{x y}$. We will find the points on the plane $\wp$ least distant from the point $A$ for the case when the area $D$ is limited and closed. If the area $D$ is not limited and closed the distance from the $A$ point to the surface $\wp_{\text {may not be the }}$ smallest among the distance values.

Suppose the point $M(x, y, f(x, y))$ is an arbitrary point on the plane ${ }^{\circ}$. In this case, the distance $S(x, y)$ between the points $A$ and $M$ can be calculated by the formula

$$
S(x, y)=\sqrt{(x-\alpha)^{2}+(y-\beta)^{2}+(f(x, y)-\gamma)^{2}} \text {. }
$$

Calculating the partial derivatives $\frac{\partial s}{\partial x}, \frac{\partial s}{\partial y}$ and equating them to zero we will get a 
system of two equations

$$
\begin{aligned}
& x-\alpha+(f(x, y)-\gamma) f_{x}^{\prime}(x, y)=0, \\
& y-\beta+(f(x, y)-\gamma) f_{y}^{\prime}(x, y)=0
\end{aligned}
$$

whose solutions are given by the abscises and ordinates of the points sought, and then by formula (13) and the corresponding distances to point $A$. Having chosen the smallest of them and from the values of the function $S(x, y)$ in the boundary points of the area $D$ we will obtain the answer to the question posed.

Assume that $\left(x_{0}, y_{0}\right)_{\text {is }}$ the solution of the system (14), corresponding to the initial distance from the point $A$ to the plane $P$. In this case, it follows that from the system (14) with $f\left(x_{0}, y_{0}\right)=z_{0}$ that

$$
\begin{gathered}
f_{x}^{\prime}\left(x_{0}, y_{0}\right)=-\frac{1}{\frac{z_{0}-\gamma}{x_{0}-\alpha}}, \\
f_{y}^{\prime}\left(x_{0}, y_{0}\right)=-\frac{1}{\frac{z_{0}-\gamma}{y_{0}-\beta}} .
\end{gathered}
$$

Consider writing an equation for a line passing through points $A$ and $M_{0}\left(x_{0}, y_{0}, z_{0}\right)$

$$
\begin{aligned}
& \frac{x-\alpha}{x_{0}-\alpha}=\frac{y-\beta}{y_{0}-\beta}=\frac{z-\gamma}{z_{0}-\gamma} \\
& \text { therefore } z-\gamma=\frac{z_{0}-\gamma}{x_{0}-\alpha}(x-\alpha) \\
& z-\gamma=\frac{z_{0}-\gamma}{y_{0}-\beta}(y-\beta) .
\end{aligned}
$$

From the comparison of the equations (15) and (16) with the equations (18) and (19), respectively, it follows thatthe tangents at the point $M_{0}$ to the flat curves obtained by crossing the surface $\wp^{\circ}$ by the planes $y=y_{0}$ and $x=x_{0}$ orthogonal line $A M_{0}$.

If the surface is set implicitly by the $F(x, y, z)=0$ equation, three $x_{k}, y_{k}, z_{k}$ numbers should be found as a solution to the system of three equations 


$$
\left\{\begin{array}{l}
(x-\alpha) \frac{\partial F}{\partial z}-(z-\gamma) \frac{\partial F}{\partial x}=0 \\
(y-\beta) \frac{\partial F}{\partial z}-(z-\gamma) \frac{\partial F}{\partial y}=0 \\
F(x, y, z)=0
\end{array}\right.
$$

and then calculate the corresponding distances $S\left(x_{k}, y_{k}\right)$ between the points $A$ and $M_{k}\left(x_{k}, y_{k}, z_{k}\right)$ and choose the smallest of them and from the values of the function $S(x, y)$ in the boundary points of the area $D$.

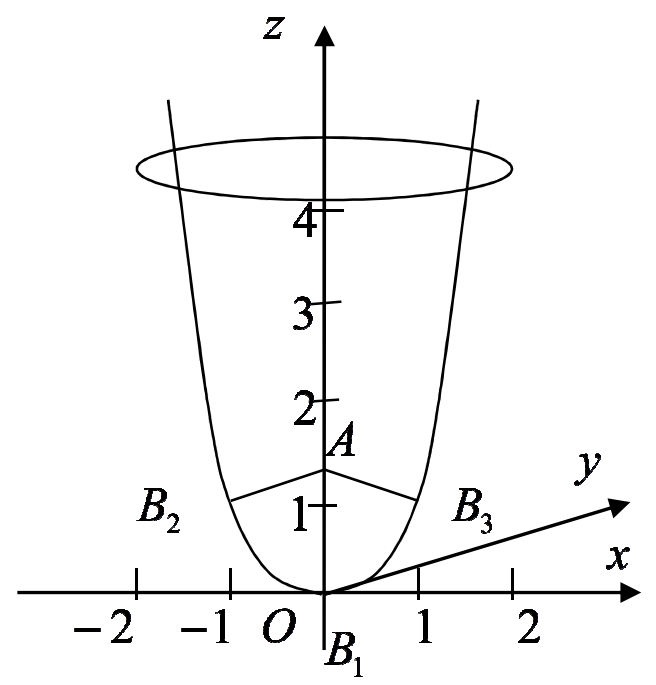

Fig. 1. Example

$$
\text { Example. On an elliptical paraboloid } z=\frac{x^{2}}{1}+\frac{y^{2}}{3} \text { it is needed to find the points lest }
$$
distant from the point $A\left(0,0, \frac{3}{2}\right)$ and determine the distance from these points to the point $A$.

Solution. Using the system equation (14), we can write

$$
\left\{\begin{array}{l}
x\left[1+\left(x^{2}+\frac{y^{2}}{3}-\frac{3}{2}\right) 2\right]=0 \\
y\left[1+\left(x^{2}+\frac{y^{2}}{3}-\frac{3}{2}\right) \frac{2}{3}\right]=0 .
\end{array}\right.
$$

The system of equations has three solutions

$$
x_{1}=0, y_{1}=0 \quad x_{2}=-1, y_{2}=0 \quad x_{3}=1, y_{3}=0 \text {. }
$$

Using the paraboloid equation, we will first find the corresponding applications, and then we will be can write (Figure.1) 


$$
B_{1}(0,0,0), \quad B_{2}(-1,0,1), \quad B_{3}(1,0,1) \text {. }
$$

For determining segment lengths $A B_{1}, A B_{2}, A B_{3}$, numbers $S_{1}, S_{2}, S_{3}$ we will use the formula (13)

$$
S_{1}=\sqrt{0^{2}+0^{2}+\left(\frac{3}{2}\right)^{2}}=\frac{3}{2}, S_{2}=\sqrt{1^{2}+0^{2}+\left(\frac{3}{2}-1\right)^{2}}=\frac{\sqrt{5}}{2}, S_{3}=\sqrt{1^{2}+0^{2}+\left(\frac{3}{2}-1\right)^{2}}=\frac{\sqrt{5}}{2},
$$

I.e. points $B_{2}$ and $B_{3}$ farthest from the point $A$.

In conclusion, we should note that the point $A$ lies at the norms of the parabola $z=x^{2}$, which is the curve resulting from the intersection of the paraboloid and the plane $y=0$, at the points $B_{2}$ and $B_{3}$. These norms have the form, respectively, of the parabola $z=\frac{1}{2} x+\frac{3}{2}$ and $z=-\frac{1}{2} x+\frac{3}{2}$.

\section{Determining the distance between a cubic parabola and a hemisphere}

Suppose that in the rectangular Cartesian system of coordinates $O x y z$ the cubic parabola $l$ and the hemisphere $S_{\text {are given respectively by the equations }}$

$$
\begin{gathered}
x_{1}=0, y_{1}=y_{1}, z_{1}=0,015 y_{1}^{3}+15, \\
z_{2}=\sqrt{36-x_{2}^{2}-y_{2}^{2}} .
\end{gathered}
$$

If selecting an arbitrary point $A\left(0, \bar{y}_{1}, \bar{z}_{1}\right)$ on the curve $l$ and plane $S_{\text {and }} B\left(\bar{x}_{2}, \bar{y}_{2}, \bar{z}_{2}\right)$ then the length of the segment $A B$ could be calculated by the formula

$$
S\left(\bar{y}_{1}, \bar{x}_{2}, \bar{y}_{2}\right)=\sqrt{\bar{x}_{2}^{2}+\left(\bar{y}_{2}-\bar{y}_{1}\right)^{2}+\left(\bar{z}_{2}-\bar{z}_{1}\right)^{2}} .
$$

Write out the necessary extremum conditions for the function $S\left(\bar{y}_{1}, \bar{x}_{2}, \bar{y}_{2}\right)$ using (20), (21) and considering $\frac{\partial S}{\partial \bar{y}_{1}}=0, \frac{\partial S}{\partial \bar{x}_{2}}=0, \frac{\partial S}{\partial \bar{y}_{2}}=0$

$$
\begin{aligned}
& \bar{y}_{2}-\bar{y}_{1}+\left(\bar{z}_{2}-\bar{z}_{1}\right) 0,045 y_{1}^{2}=0, \\
& \bar{x}_{2}+\left(\bar{z}_{2}-\bar{z}_{1}\right)\left(-\frac{\bar{x}_{2}}{\bar{z}_{2}}\right)=0,
\end{aligned}
$$




$$
\bar{y}_{2}-\bar{y}_{1}+\left(\bar{z}_{2}-\bar{z}_{1}\right)\left(-\frac{\bar{y}_{2}}{\bar{z}_{2}}\right)=0 .
$$

It is evident that if we consider $\bar{x}_{2}=0$, then the system of equations (23), (24), (25) have solutions

$$
\bar{x}_{2}=0, \quad \bar{y}_{1}=0, \quad \bar{y}_{2}=0
$$

and then from equations (20) and (21) follows

$$
\bar{z}_{1}=15, \quad \bar{z}_{2}=6,
$$

and therefore, in accordance with equation (22), we are able to write

$$
S(0,0,0)=9
$$

therefore $A(0,0,15)$, and $B(0,0,6)$.

Considering, that $\bar{x}_{2} \neq 0$, it follows from the equation (5) that $\bar{z}_{1}=0$, then from equation (25) it follows that $\bar{y}_{1}=0$, and from equation (20), that $\bar{y}_{1}=-10$.

That means $\bar{x}_{2}=0$.

Calculating $\bar{x}_{2}=0$, we will look for other solutions (different from (26)) to the system of two equations (23), (25).

From a comparison of these equations, it follows that

$$
-\bar{y}_{2}=0,045 \bar{y}_{1}^{2} \bar{z}_{2} \text {. }
$$

Equation (25) can be written as

or, using equation (29),

$$
\bar{y}_{2}-\bar{y}_{1}-\bar{y}_{2}-\bar{z}_{1}\left(-\frac{\bar{y}_{2}}{\bar{z}_{2}}\right)=0
$$

$$
\bar{y}_{1}+0,045 \bar{y}_{1}^{2} \bar{z}_{1}=0
$$

If we take $\bar{y}_{1}=0$ in the equation (30), the it follows from the equation (23) that $\bar{y}_{2}=0$ , i.e., the solution (26) is obtained.

Considering the case when $\bar{y}_{1} \neq 0$. Then, dividing both parts of the equality (30) by $\bar{y}_{1}$ and using equality $\bar{z}_{1}$ of (20), we obtain the equation to determine $\bar{y}_{1}$

$$
6,75 \cdot 10^{-4} \bar{y}_{1}^{4}+0,675 \bar{y}_{1}+1=0 .
$$

This equation has two tangible roots: 


$$
\bar{y}_{11}=-9,447, \quad \bar{y}_{12}=-1,486
$$

and two complexes $5,467 \pm 8,696 i$.

Using formula (20) we will find the corresponding values

$$
\bar{z}_{11}=2,353, \quad \bar{z}_{12}=14,951 \text {. }
$$

Substituting sequentially the value of $\bar{y}_{11}$ and $\bar{y}_{12}$ in the equation (29), we will get

$$
\begin{aligned}
& -y_{21}=4,016 \bar{z}_{21}, \\
& -y_{22}=0,099 \bar{z}_{22} .
\end{aligned}
$$

From equation (21) at $\bar{x}_{2}=0$ it follows that

$$
\bar{z}_{2}^{2}=36-\bar{y}_{2}^{2} \text {. }
$$

First, we will write out the distance between the points $A_{1}(-9,447 ; 2,353)$ and $B_{1}(-5,823 ; 1,45)$, then between points $A_{2}(-1,486 ; 14,951)$ and $B_{2}(-0,591 ; 5,97)$

$$
\begin{aligned}
& S_{1}=\sqrt{3,624^{2}+0,903^{2}}=3,734, \\
& S_{2}=\sqrt{0,895^{2}+8,981^{2}}=9,025 .
\end{aligned}
$$

A comparison of three numbers from (28), (37) and (38) reveals that the distance required is 3,734 (see Figure 2). 


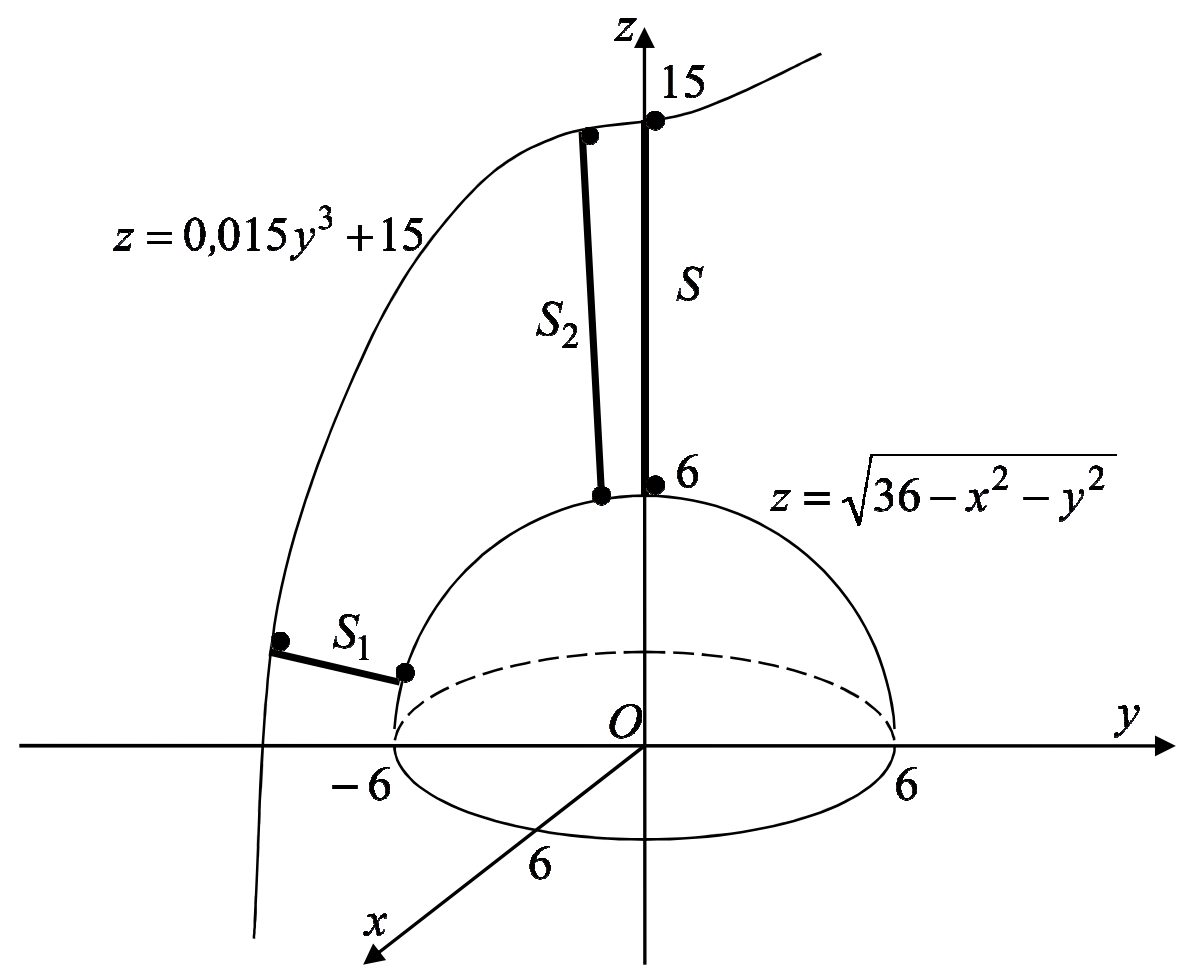

Fig. 2. Result

\section{References}

1. L. Bakeeva et al., Atlantis Highlights in Computer Sciences, 1, 257-261 (2019). https://doi.org/10.2991/icdtli-19.2019.46 